\title{
Differential distribution of a SINE element in the Entamoeba histolytica and Entamoeba dispar genomes: Role of the LINE-encoded endonuclease
}

Vandana Kumari ${ }^{1 \dagger}$, Rahul Sharma $^{1 \dagger}$, Vijay P Yadav ${ }^{1}$, Abhishek K Gupta $^{1}$, Alok Bhattacharya ${ }^{2}$ and Sudha Bhattacharya ${ }^{1 *}$

\begin{abstract}
Background: Entamoeba histolytica and Entamoeba dispar are closely related protistan parasites but while E. histolytica can be invasive, E. dispar is completely non pathogenic. Transposable elements constitute a significant portion of the genome in these species; there being three families of LINEs and SINEs. These elements can profoundly influence the expression of neighboring genes. Thus their genomic location can have important phenotypic consequences. A genome-wide comparison of the location of these elements in the E. histolytica and E. dispar genomes has not been carried out. It is also not known whether the retrotransposition machinery works similarly in both species. The present study was undertaken to address these issues.
\end{abstract}

Results: Here we extracted all genomic occurrences of full-length copies of EhSINE1 in the E. histolytica genome and matched them with the homologous regions in E. dispar, and vice versa, wherever it was possible to establish synteny. We found that only about $20 \%$ of syntenic sites were occupied by SINE1 in both species. We checked whether the different genomic location in the two species was due to differences in the activity of the LINE-encoded endonuclease which is required for nicking the target site. We found that the endonucleases of both species were essentially very similar, both in their kinetic properties and in their substrate sequence specificity. Hence the differential distribution of SINEs in these species is not likely to be influenced by the endonuclease. Further we found that the physical properties of the DNA sequences adjoining the insertion sites were similar in both species.

Conclusions: Our data shows that the basic retrotransposition machinery is conserved in these sibling species. SINEs may indeed have occupied all of the insertion sites in the genome of the common ancestor of E. histolytica and E. dispar but these may have been subsequently lost from some locations. Alternatively, SINE expansion took place after the divergence of the two species. The absence of SINE1 in $80 \%$ of syntenic loci could affect the phenotype of the two species, including their pathogenic properties, which needs to be explored.

\section{Background}

Transposable elements are found in the genomes of almost all organisms, and are of ancient origin. Their ability to insert into new genomic locations makes them potent agents of phenotypic change, including various known pathologies [1-3]. Transposons are also found in

\footnotetext{
* Correspondence: sbjnu110@gmail.com

† Contributed equally

'School of Environmental Sciences, Jawaharlal Nehru University, New Delhi

110067, India

Full list of author information is available at the end of the article
}

parasites, for example the human enteric pathogen, Entamoeba histolytica, a unicellular eukaryote contains three families of the autonomous non long terminal repeat (LTR) retrotransposon called EhLINE and its nonautonomous partner EhSINE [4-8]. The genome of the morphologically indistinguishable sibling species Entamoeba dispar, (which resides in the human colon but is nonpathogenic), also contains three families of EdLINEs and their partner EdSINEs [6,7,9]. It would be of interest to know whether these retrotransposons have any influence on pathogenesis-related gene expression.
C Biomed Central

() 2011 Kumari et al; licensee BioMed Central Ltd. This is an Open Access article distributed under the terms of the Creative Commons Attribution License (http://creativecommons.org/licenses/by/2.0), which permits unrestricted use, distribution, and reproduction in any medium, provided the original work is properly cited. 
LINEs and SINEs are known to influence the expression of neighboring genes by a variety of mechanisms, for example by providing alternative promoters, splicing and polyadenylation sites and by heterochromatinization [10-13]. For this reason it is important to investigate whether these elements are located at syntenic positions in the E. histolytica and E. dispar genomes. Earlier investigations with a limited number of genomic loci showed that the sites occupied by EhSINE1 in the $E$. histolytica genome were empty at homologous regions in E. dispar [14] and conversely the sites occupied by EdSINE1 in the E. dispar genome were empty in $E$. histolytica [9]. Although an exhaustive genome-wide survey of LINEs and SINEs in Entamoeba species has been reported [6], a genome-wide comparison of the occupancy of these elements at syntenic loci in E. histolytica and $E$. dispar has not been carried out. Here we present results of a genome-wide comparison of EhSINE1 and EdSINE1 in the two genomes. In addition we address the question whether the differences in genomic locations of retrotransposons in these two organisms could be due to inherent differences in the retrotransposition machinery, particularly in the properties of the LINEencoded endonuclease. Target primed reverse transcription is the mechanism by which non-LTR retrotransposons insert in the genome [15]. Since retrotransposition is initiated by the element-encoded endonuclease (EN) making a nick at the bottom strand of the site of insertion, an important determinant of target site specificity could be the preferred nucleotide sequences recognized by the EN. We have earlier shown that the EhLINE1encoded EN (Eh EN) nicks preferentially at the consensus sequence 5'-GTATT-3', between A-T and T-T. Here we have investigated whether the endonuclease domain encoded by EdLINE1 (Ed EN) has a different target site specificity which could account for the lack of synteny in the location of SINEs in the two genomes.

\section{Methods}

\section{Comparative Analysis of E. histolytica and E. dispar with respect to the occupancy of SINE1 elements}

Since the Entamoeba genome has not yet been assembled completely for any species, we have done all of our comparative analysis on the basis of SINE1 elements located on the scaffolds. The genome sequence of $E$. histolytica having 1529 scaffolds and $E$. dispar having 12258 scaffolds was downloaded [NCBI:AAFB00000000, NCBI:AANV00000000]. A total of 393 EhSINE1 and 302 EdSINE1 elements having lengths greater than 450 bp were located. The EhSINE1 sequences were from Huntley et al [4] and the EdSINE1 sequences were from the feature table file of $E$. dispar (updated on Dec. 8, 2008). For genes flanking the EhSINE1 sequences the feature table file of E. histolytica (updated on April 17,
2008) was used. These were downloaded from NCBI and the genes upstream and downstream of SINE1 elements in each of the species were located using perl coding. Finally the orthologues of these genes were searched in the other species using BLAST [16]. The syntenic loci were checked to see whether SINE1 was present there in both species. To check the homology at Scaffold level we used GATA, a graphic alignment tool for comparative sequence analysis [17]. Syntenic loci were further examined to check for the presence of SINE in both species.

DNA sequence features of the SINE1 insertion sites were computed by extracting flanking 80 bp sequences (40 bp upstream and downstream) from the respective genomic sites in E. histolytica and E. dispar, using perl scripting. "DNA SCANNER" was used as previously described [18] to calculate the DNA parameters, which include $T$ rule, bendability, propeller twist, stacking energy, duplex stability, DNA denaturation, proteininduced deformability, nucleosomal positioning and bending energy [18].

\section{Construction and Cloning of Ed EN}

The consensus amino acid sequences of the E. dispar and $E$. histolytica endonuclease domains, Ed EN and Eh EN respectively were compared. A total of 23 amino acid positions were different of which 12 amino acid residues were changed in the Eh EN sequence [19] and 11 amino acid residues (synonymous) were left unchanged to obtain the Ed EN sequence. The desired mutations were introduced either by means of overlapping PCR (Additional file 1 Figure S1) or by site-directed mutagenesis. The primer pairs used are listed (Additional file 2 Table S1). The Ed EN sequence thus obtained (782 bp) was cloned into the EcoRI-NotI site of pET30(b) vector (Novagen) to yield the pET-Ed-EN construct.

\section{Expression and purification of recombinant Ed EN}

This was done essentially as described for Eh EN [19]. The pET-Ed-EN construct was transformed in Escherichia coli BL-21 (DE3). Cells were grown in $200 \mathrm{ml}$ of Luria Broth at $30^{\circ} \mathrm{C}$ to $\mathrm{OD}_{600}$ of 0.6 . For induction of hexa-His-tagged Ed EN, IPTG was used to a final concentration of $0.5 \mathrm{mM}$ and the cells were further incubated for 2 hour. The protein was purified by $\mathrm{Ni}^{2}$ ${ }^{+}$-nitrilotriacetic acid-agarose (Qiagen) affinity chromatography and eluted with $250 \mathrm{mM}$ of imidazole. The eluted fractions were checked for the protein by resolving on a $12 \%$ SDS PAGE; these fractions were pooled, dialyzed and stored at $-20^{\circ} \mathrm{C}$. The enzyme activity of $\mathrm{Ed}$ EN was measured under the same conditions used for Eh EN, i.e. $\mathrm{pH} 7.0$, at $37^{\circ} \mathrm{C}$, and at $\mathrm{Mg}^{2+}$ and $\mathrm{NaCl}$ concentrations of 10 and $100 \mathrm{mM}$ respectively [20]. 


\section{Western blotting}

The whole cell lysate of induced and uninduced BL-21 (DE3) cells was resolved on 12\% SDS PAGE and transferred to PVDF membrane by semidry transfer method according to manufacturer's instructions (Bio-Rad). The membrane was blocked overnight with $5 \%$ skimmed milk in PBS-T (Phosphate buffer saline with $0.1 \%$ Tween 20) and subsequently incubated with anti-His antibody or anti-Eh EN antibody for 1 hour followed by three times washing with PBS-T. The membrane was further incubated with horseradish peroxidase-conjugated secondary antibody and again washed with PBS-T thrice. The protein was detected by the Chemiluminescent HRP Substrate (Millipore).

\section{Nicking assay of radiolabeled 176 bp substrate}

The 176 bp DNA fragment was radiolabeled as described earlier [19]. The labeled DNA was resolved on $6 \%$ native gel, the band was visualized by ethidium bromide staining, excised from the gel and eluted by crush and soak method [21]. The nicking assay was carried out as described for Eh EN and products were resolved by denaturing electrophoresis on $6-8 \%$ polyacrylamide gels containing $7 \mathrm{M}$ Urea [19]. The gels were dried and autoradiographed in the PhosphorImager (Fujifilm).

\section{Nicking assay of pBS DNA}

Supercoiled pBluescript (pBS) plasmid DNA was purified by plasmid purification kit (Qiagen). $2 \mathrm{nM}$ of purified Ed EN was incubated with 2-75 $\mathrm{nM}$ of supercoiled pBS DNA. The reaction was performed under the conditions employed for Eh EN [20], at $37^{\circ} \mathrm{C}$ for 8 minutes and was stopped with $25 \mathrm{mM}$ EDTA. Products were separated on $0.8 \%$ agarose gel containing $0.5 \mu \mathrm{g}$ of ethidium bromide $/ \mathrm{ml}$. Under this condition the supercoiled DNA migrated fastest, followed by linear and open circular form. The intensity of supercoiled DNA band was measured as a function of time, which gave the measure of the disappearance of supercoiled DNA. Quantification was done by densitometry; the kinetic constants $V_{\text {max }}$, $K_{m}$ and $k_{c a t}$ were determined as described [22]. The data obtained were the average of three independent determinations.

\section{Results and Discussion}

Comparative analysis of EhSINE1-containing regions of $E$. histolytica genome and syntenic regions of $E$. dispar

A total of 393 full-length SINE1 elements (length $>450$ bp) were identified in E. histolytica by genome sequence analysis. Syntenic regions corresponding to each of the EhSINE1-containing loci were located in E. dispar. To score for synteny entire scaffolds were matched in the two species using the program GATA. In $88 \%$ of cases where synteny was found, the syntenic regions matched throughout the scaffold, while in the rest synteny was not visible in some patches. The presence or absence of any of the EdSINEs (EdSINE1, 2, 3) was determined at syntenic loci of $E$. dispar. The results are summarized in Figure 1A. Of the 393 EhSINE1-containing loci of $E$. histolytica, syntenic regions in E. dispar could be predicted with certainty for 180 loci. Only these loci were included for further study - thus removing the contribution of differential sequence coverage in our comparative analysis. Loci not represented in both species due to differences in genome coverage, or difficulty in alignment, have not been included. In addition, as stated above, the synteny stretched for the entire length of the scaffold. Of these 180 loci, SINEs were absent in E. dispar at 114 loci, were present at 24 loci and their presence or absence could not be determined at 42 loci. Amongst the 114 loci where SINEs were absent, no repeat element of any type was found at 96 loci (representative example shown in Additional file 3 Figure S2), while LINE or EdRC4 sequences were found at 18 loci. Amongst the 24 loci that contained a SINE, 18 had EdSINE1 (one representative example is shown in Figure 2 and the rest in Additional file 4 Figure S3-S19), one had a truncated copy of EdSINE1, and five had EdSINE2 and/or EdSINE3. Amongst the 42 loci where presence or absence of SINE could not be established, in 23 cases the scaffold ended within the locus in E. dispar, and in 19 cases the homology was restricted to genes on one side of the SINE while there was no homology on the other side (may be due to deletions/inversions/ rearrangements).

For 213 EhSINE1-containing loci of E. histolytica, syntenic loci could not be found in E. dispar for the following reasons. In many of these cases the scaffolds containing these loci were composed entirely of repeats (83 loci), tRNA genes and repeats (7 loci) or pseudogenes and repeats (9 loci). In 114 cases synteny could not be determined either because there were multiple copies of homologous genes, or homologous genes were located on multiple scaffolds, or there was a single gene in the scaffold.

\section{Comparative analysis of EdSINE1-containing regions of $E$.} dispar genome and syntenic regions of $E$. histolytica A total of 302 full-length SINE1 elements (length greater than $450 \mathrm{bp}$ ) were identified in $E$. dispar by genome sequence analysis. Syntenic regions corresponding to each of the EdSINE1-containing loci were located in $E$. histolytica and the presence or absence of any of the EhSINEs (EhSINE1, 2, 3) was determined as described above. Of the 302 loci, syntenic regions could be predicted with certainty for 127 loci (Figure 1B). Of these, SINEs were absent in E. histolytica at 73 loci, were present at 19 loci and their presence or absence could not be determined at 35 loci. Amongst the 73 loci where 


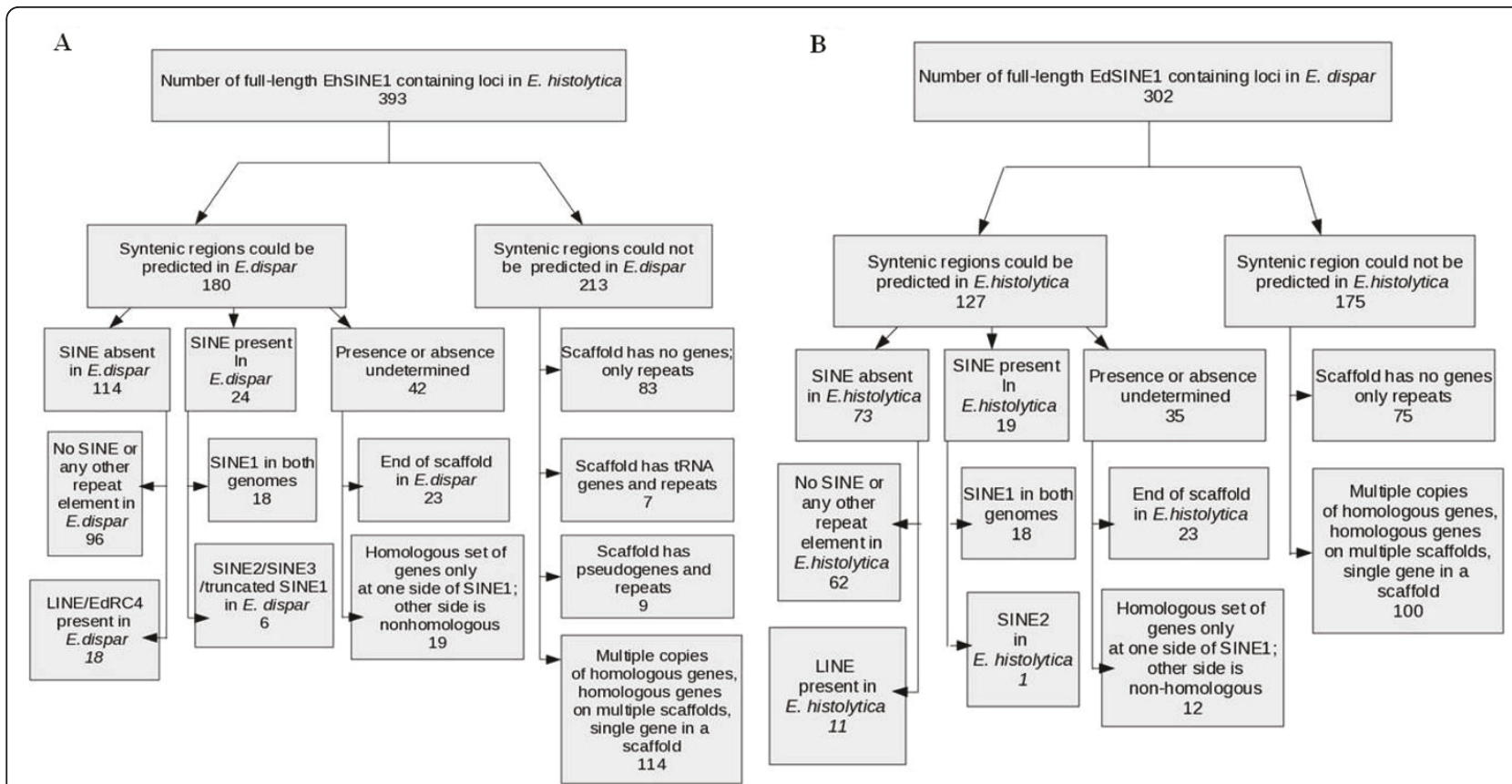

Figure 1 Summary of the comparative analysis of genomic locations of SINE1 copies in E. histolytica and E. dispar. (A). Comparison of EhSINE1-occupied regions in E. histolytica genome with syntenic regions of E. dispar. (B). Comparison of EdSINE1-occupied regions in E. dispar genome with syntenic regions of E. histolytica.

SINEs were absent, no repeat element of any type was found at 62 loci (Additional file 5 Figure S20), while LINE sequences were found at 11 loci. Amongst the 19 loci that contained a SINE, 18 had EhSINE1 (as scored above) while 1 had EhSINE2. Amongst the 35 loci where presence or absence of SINE could not be established, in 23 cases the scaffold ended within the locus in E. histolytica, and in 12 cases the homology was restricted to genes on one side of the SINE while there was no homology on the other side.

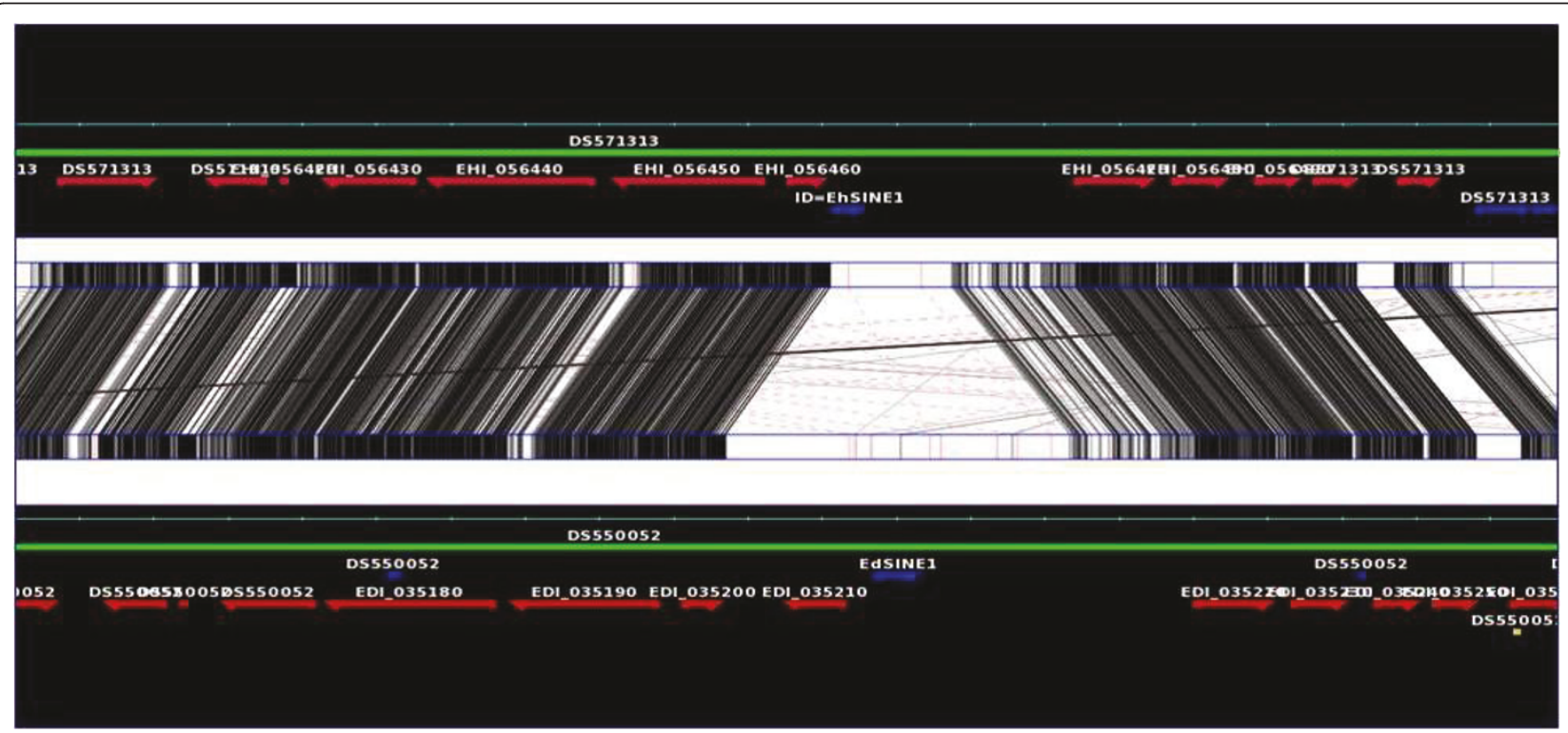

Figure 2 Graphical representation of a syntenic region where SINE1 is present in both E. histolytica and E. dispar. Top and bottom green lines mark the genomes of E. histolytica and E. dispar respectively. The E. histolytica and E. dispar genes are marked by red arrows, showing the gene orientation. Shaded black lines showing the identical region within E. histolytica and E. diapar. Darkness of the shade is proportional to the \% identity, with white being the least conserved. Blue arrows show the repeat elements (EhSINE1 and EdSINE1). 
For 175 EdSINE1-containing loci, syntenic loci could not be found in E. histolytica for the reasons mentioned in the previous section. In 75 cases the $E$. dispar loci were composed entirely of repeats while in 100 cases synteny could not be determined either because there were multiple copies of homologous genes, or homologous genes were located on multiple scaffolds, or there was a single gene in the scaffold.

\section{Sequence alignment of syntenic loci}

Figure 3 shows actual sequence alignments of a few selected syntenic loci where SINE1 is found in E. histolytica but missing in E. dispar, and vice versa. As is evident, in each case the element is flanked by TSDs. Only one copy of the TSD is found at the syntenic locus of the species where the SINE is missing. The surrounding sequences show the sequence similarity expected of

\section{A}

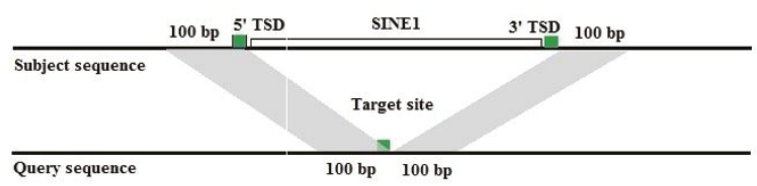

Subject: E. histolytica; Query: E.dispar

Locus 1

Eh $D \$ 571347.1$

Ed DS550300.1

Eh $D 5571347.1$

Ed DS550300.1

Locus 2

Eh 05571257.1

Ed $0 \$ 547864.1$

Eh 05571257.1

Ed 05547864.1

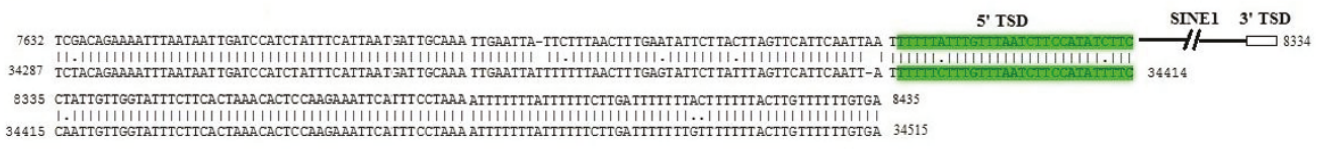

B
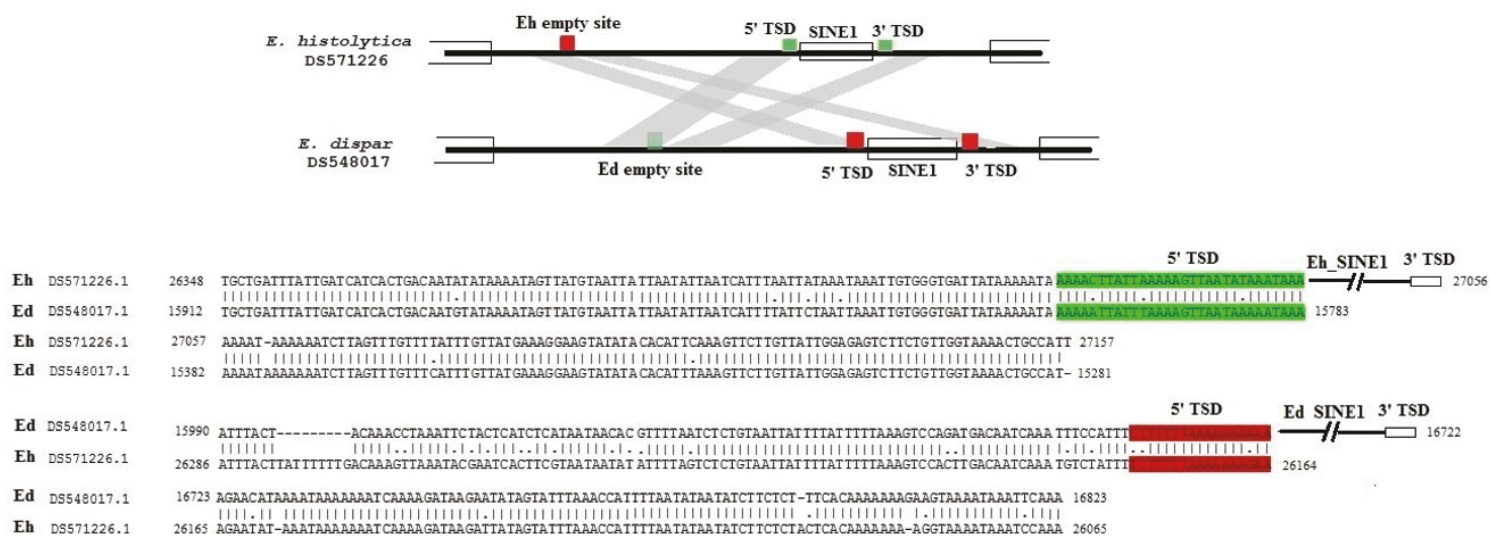

Figure 3 Sequence alignment of syntenic intergenic regions. (A) SINE1 is absent in one of the species; (B) SINE1 is present in both species. The scaffold number and nucleotide positions are indicated. TSD, target site duplication. 
intergenic regions of the two species (80-90\%). One example is shown of an intergenic region where SINE1 is present in both species. Although SINE1 is located in the same intergenic region, the actual point of insertion is not the same and consequently the TSD sequences are different (Figure 3B). This was the typical pattern seen in other loci of this type where SINEs were located in the same intergenic regions in both species.

From the above data it is clear that only in about $20 \%$ of cases where presence or absence of SINE1 could be established at syntenic loci, are SINE elements located in the same intergenic region (although at different insertion points) in both species. In $>80 \%$ of these loci SINE1 was not found at the same location in both species. Since the elements in the two species have a common lineage and are closely related, what possible factors might account for these differences? According to the Target primed Reverse Transcription model, retrotransposition is initiated by the LINE-encoded Endonuclease (EN) nicking the bottom strand of the target site [15]. Hence it is reasonable to believe that the sequences preferentially nicked by the EN could be the preferred insertion sites of the retrotransposon, and the behavior of EN might influence the choice of target site of a non LTR retrotransposon. Since the Eh EN and Ed
EN differ from each other at many amino acid positions (as shown below), it is possible that the two enzymes may have evolved different recognition specificities. To establish this we studied the properties of the EdLINE1encoded EN and compared it with EhLINE1-encoded EN.

\section{Cloning and expression of the EdLINE1 endonuclease (Ed EN) polypeptide}

To obtain the Ed EN coding sequence we used the Eh EN sequence (already cloned in our lab) as a starting point. Ed EN differs from Eh EN in 23 amino acid positions (Figure 4). The Eh EN sequence was mutated in these positions (as described in Methods) to obtain the Ed EN coding sequence. This 782-bp EcoRI-Not1 fragment was cloned in the $E$. coli expression vector pET30b. The expressed protein contained His-tag, and together with other vector sequences at the amino terminus, it was 307 amino acids long, with an expected molecular mass of $35.3 \mathrm{kDa}$ (Figure 5A). It was purified by nickel-agarose chromatography, and its identity was confirmed by using an anti-His tag antibody and anti-Eh EN antibody (Figure $5 B)$. The Ed EN protein could nick a nonspecific substrate, pBS. Like the previously reported activity of Eh EN [19] supercoiled pBS DNA was efficiently nicked by

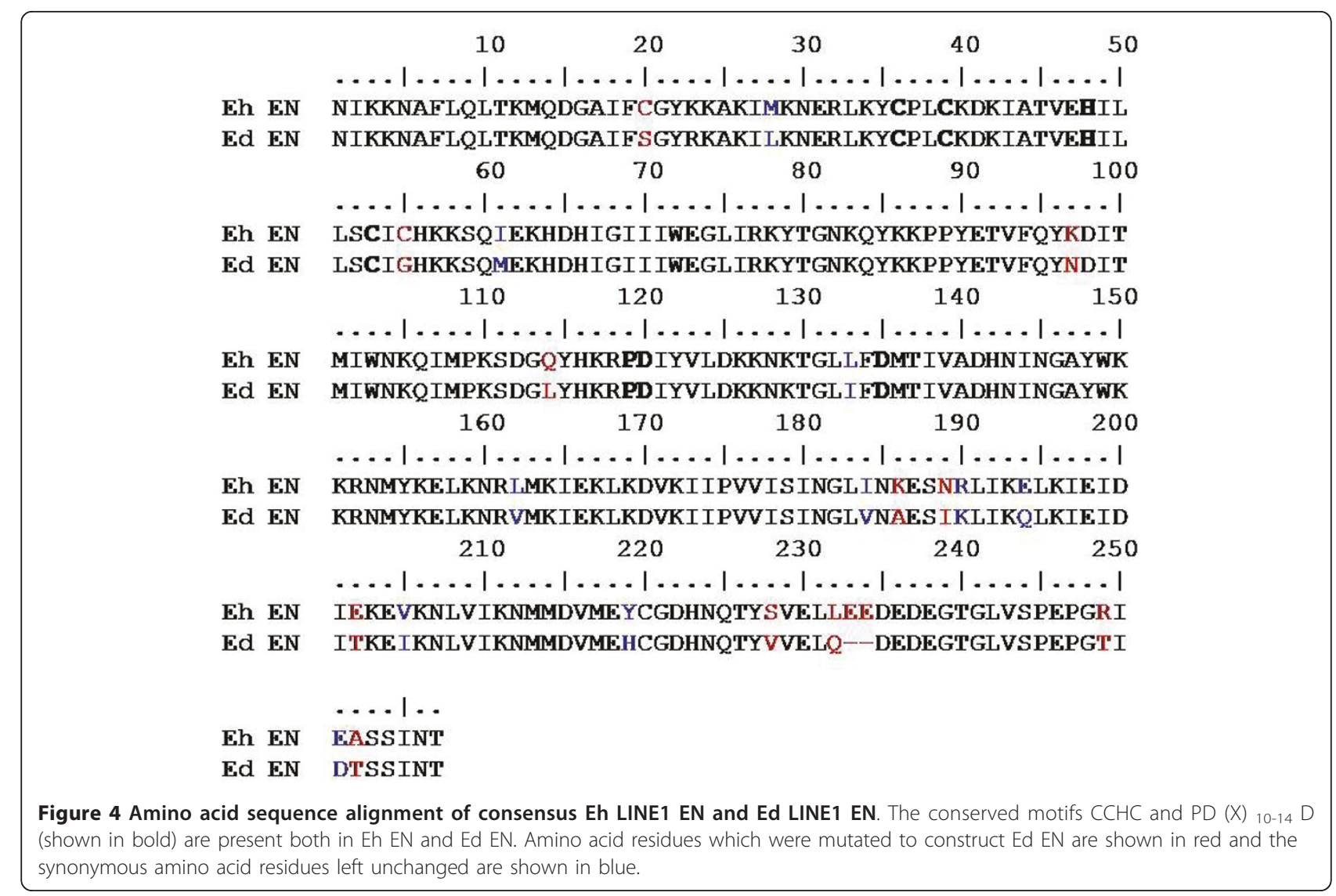




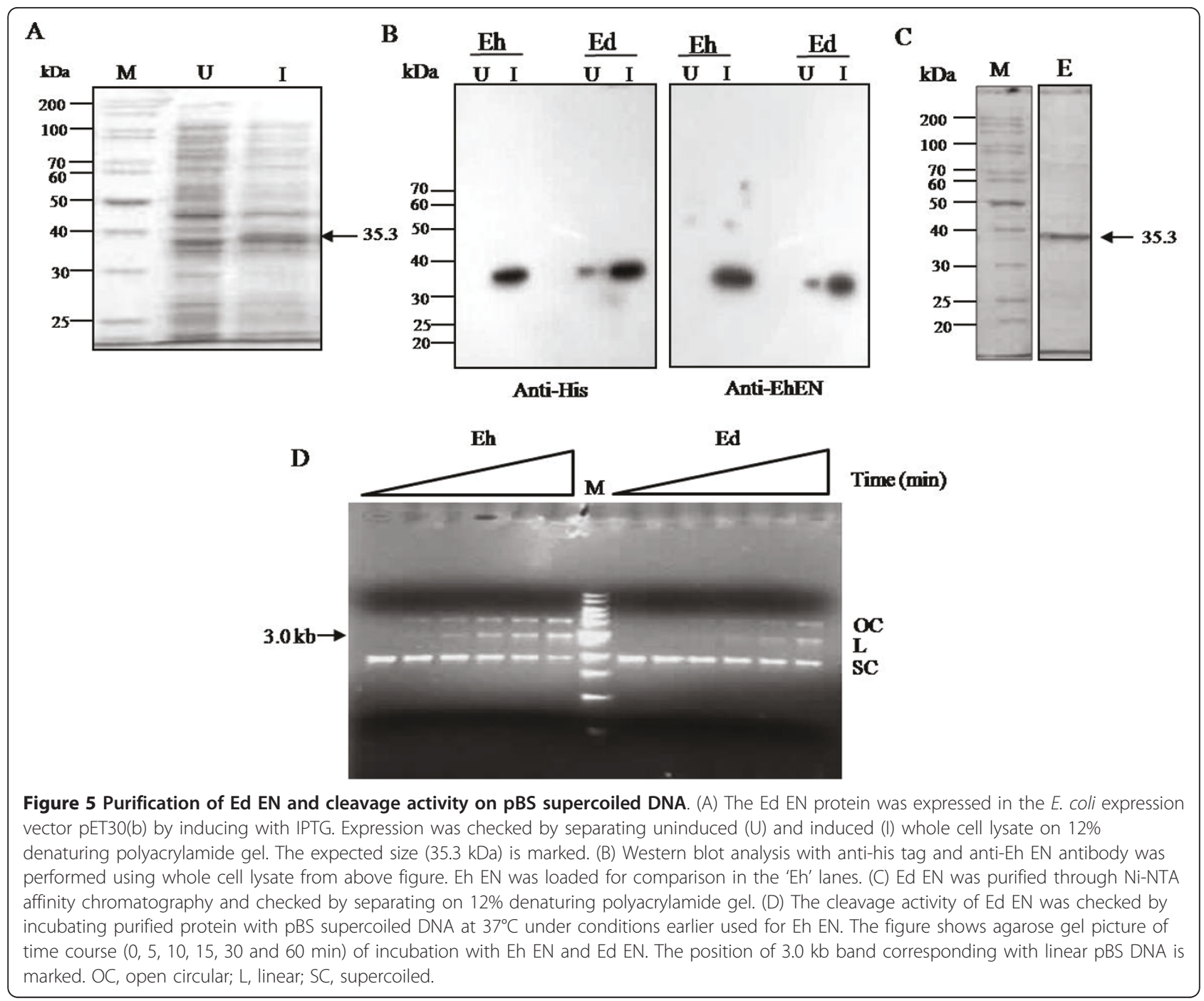

the purified Ed EN protein to yield open circular and linear DNAs. The presence of discrete bands corresponding to open circular and linear forms shows that the enzyme makes predominantly single-strand nicks and not double-strand breaks (Figure 5D).

\section{Kinetics of the Ed EN-catalyzed reaction with pBS} supercoiled DNA substrate under steady-state conditions To determine the kinetics under steady-state conditions, reactions were carried out with the enzyme at a concentration of $2 \mathrm{nM}$ and with pBS DNA at a concentration of 2-75 nM (Figure 6). The disappearance of supercoiled DNA was determined by densitometric scanning as described for Eh EN [20]. As mentioned in Methods, all time-course results were the average of at least three independent determinations. The variation observed at each time point was $<4.7 \%$ of the mean value (0.09-5.1). Although the variation in values of each data point was in the range of $4 \%$ in three replicates, the slopes for each set showed lesser variation (up to 1.0\%). Kinetic parameters $\left(\mathrm{K}_{\mathrm{m}}\right.$ and $\left.\mathrm{k}_{\mathrm{cat}}\right)$ were calculated from a Lineweaver-Burk plot (Figure $6 \mathrm{C}$ ). $\mathrm{K}_{\mathrm{m}}$ for $\mathrm{pBS}$ DNA was calculated to be $1.086 \pm 0.009 \times 10^{-8} \mathrm{M}$. The catalytic constant, $\mathrm{k}_{\text {cat }},\left(\mathrm{V}_{\max } /[\mathrm{E}]\right)$ was determined to be $5.67 \pm$ $0.027 \times 10^{-3} \mathrm{sec}^{-1}$. These values were comparable to the $\mathrm{K}_{\mathrm{m}}\left(2.6 \pm 0.018 \times 10^{-8} \mathrm{M}\right)$ and $\mathrm{k}_{\text {cat }}\left(1.6 \pm 0.0110^{-2} \mathrm{sec}^{-}\right.$ $\left.{ }^{1}\right)$ of Eh EN [20] and the $K_{m}$ was comparable with the low $\mathrm{K}_{\mathrm{m}}$ values (0.5-17 $\mathrm{nM}$ ) of restriction endonucleases determined with different DNA substrates under different conditions of buffer and temperature [22]. Furthermore, the turnover number $\left(k_{\text {cat }}\right)$ of the enzyme was in the lower range of that reported for restriction endonucleases [(1.6-16.6) $\left.\times 10^{-2} \mathrm{sec}^{-1}\right]$ [22]. The low turnover number of a retrotransposon-encoded endonuclease may have a significant role in limiting the rate of retrotransposition events in the genome. From the above data we infer that the kinetic parameters of Ed EN are not significantly different from Eh EN. 


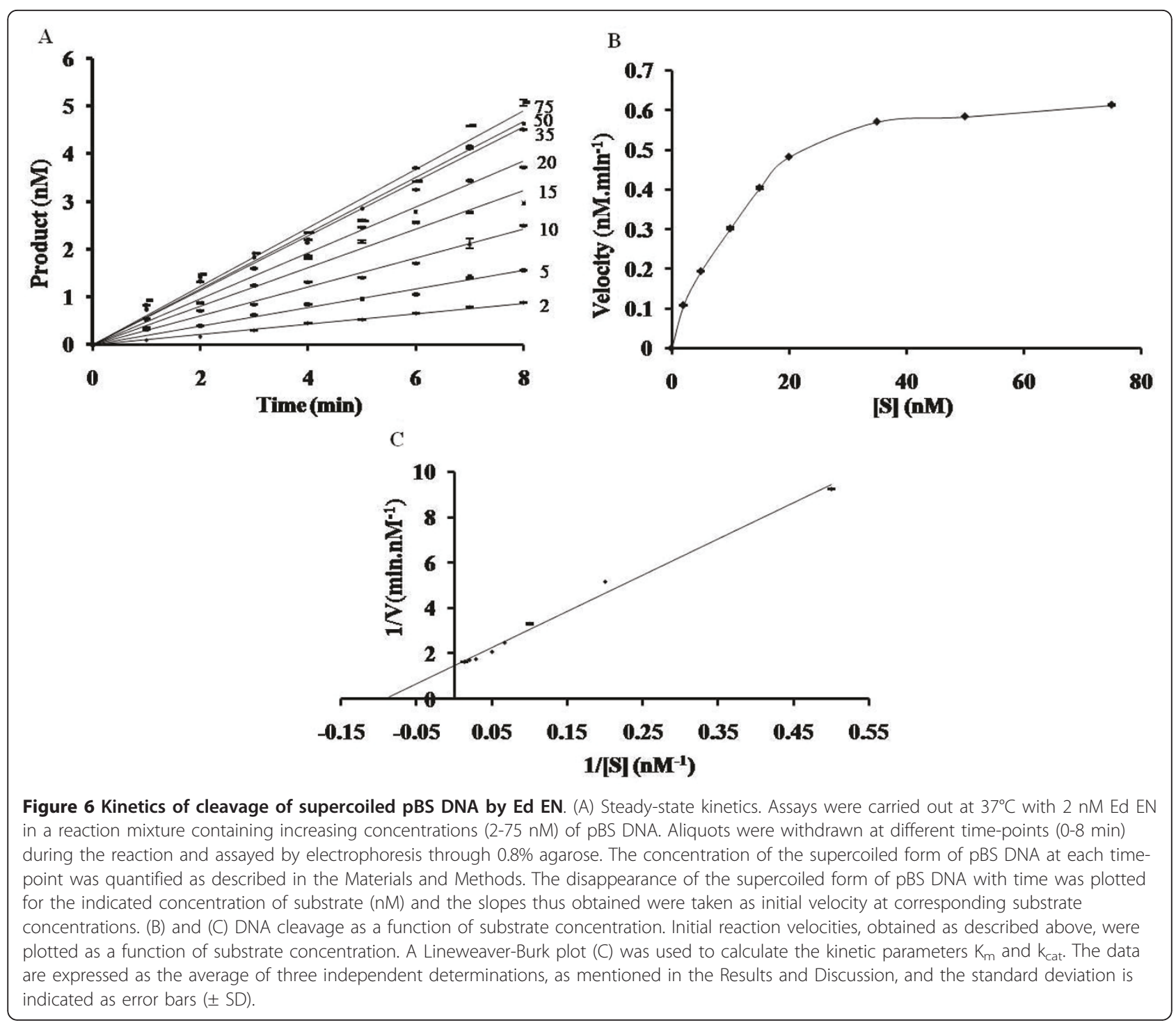

\section{Nicking site sequence preference of Ed EN}

We had earlier shown that Eh EN preferentially nicked a $176 \mathrm{bp}$ fragment from $E$. histolytica precisely at the site where a SINE1 element was known to insert in this region of the genome [19]. To test whether Ed EN had a similar sequence preference as Eh EN, the same $176 \mathrm{bp}$ fragment was incubated with Ed EN. The nicking pattern, determined for the bottom strand, was exactly the same as that obtained with Eh EN. Three nicking hot spots were obtained, of which site \#3 corresponded with the exact site of insertion of Eh SINE1 in vivo (Figure 7A). The sequences important for target site recognition, as determined for Eh EN [18], were tested for Ed EN by altering the sequences immediately surrounding the nicked site \#3. Transition mutations were introduced using oligonucleotides with the appropriately altered sequence to PCR amplify a $117 \mathrm{bp}$ fragment from the 176 bp template (position 60 to 176). The DNA sequences thus obtained contained a normal site \#2 and a mutated site \#3. The activity of Ed EN on the mutated site \#3 was quantitated using site \#2 as an internal control. The results showed that changing the GG nucleotides (on top strand, upstream of the nick) to TT decreased the activity to 10\% for Ed EN (compared with $2 \%$ for Eh EN) and changing the T nucleotide upstream of the nick to $C$ increased the activity to $183 \%$ for Ed EN (compared with 133\% for Eh EN) (Figure 7B). These results show that both Ed EN and Eh EN are very similar in their target site specificity. Since the endonuclease domain of ORF2 was used in these studies, the possibility of a complete ORF2 protein displaying a different specificity in vivo cannot, however, be entirely ruled out. 


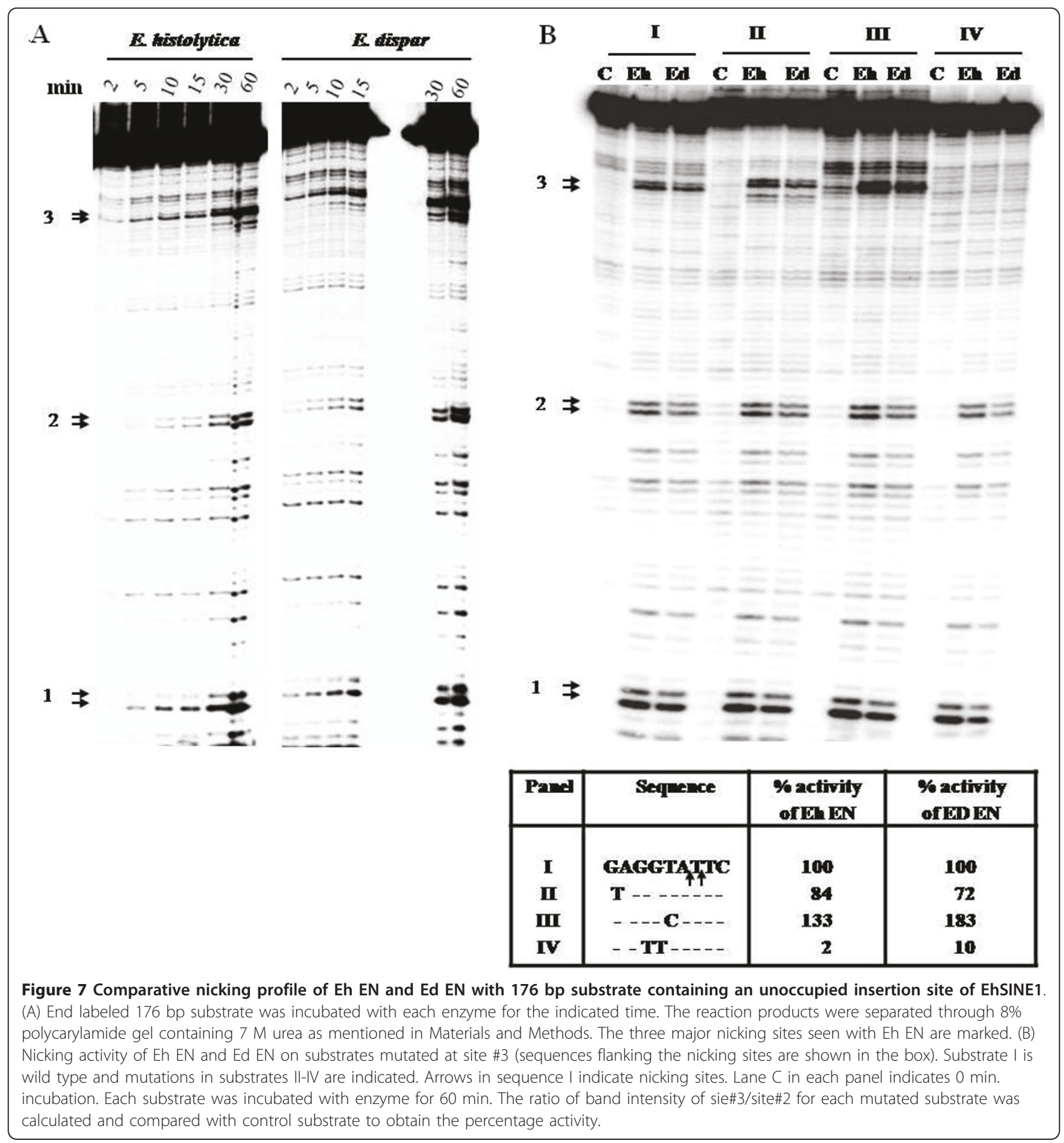

\section{DNA structural features of E. dispar and E. histolytica}

SINE1 insertion sites

We have earlier shown that sequence-dependent DNA structural features may play an important role in site selection by SINE elements in E. histolytica [18]. Here we have analyzed the same features in E. dispar to see if the SINE insertion sites share these properties with $E$. histolytica. The parameters checked are listed in 'Methods'. In general majority of the features showed similar pattern in both the species except for DNA denaturation energy and free energy profile. Our previous study had shown that the insertion sites in E. histolytica are Tenriched and the content profile showed a significant peak at -22 bp relative to the insertion site. Similar profile was also observed for E. dispar with a peak at -22 bp (Figure 8). Statistical analysis by Mann-Whitney test on the difference of the average $\mathrm{T}$ content of insertion sites for E. histolytica and E. dispar suggested that there 


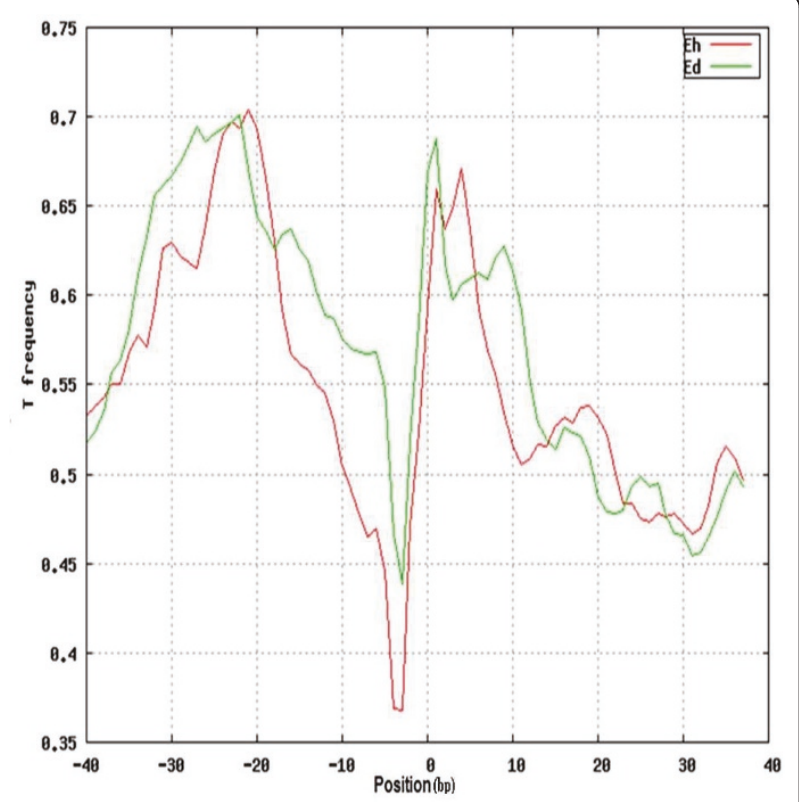

Figure 8 T content profile. Profile shows the frequency of $T$ residues flanking the SINE1 insertion sites. Details are provided in Materials and Methods. Both E. histolytica (red) and E. dispar (green) show high $T$ content at -22 position.

is no significant difference between the two. Similar results were obtained when other DNA-based structural parameters were determined. Thus the SINE1-occupied sites in E. histolytica and E. dispar share the same structural features.

We checked whether the intergenic regions at loci where SINEs were found in both genomes shared greater sequence similarity compared with loci where SINEs did not occur in both genomes. However this was not found to be the case. Intergenic regions in both sets of loci showed overall sequence similarity in the range of $75-90 \%$. E. histolytica and $E$. dispar are very closely related sibling species [23] which were in fact classified as a single species until they were re-described as two separate species [24]. Their close relationship is also evident from phylogeny based on LINE-derived RT sequences. This analysis showed that all three families of LINEs and SINEs already existed in the common ancestor before E. histolytica and E. dispar separated into two distinct species [6]. The great similarity between the two ENs of E. histolytica and E. dispar, as found in this study, again shows that the basic retrotransposition machinery is highly conserved in these sibling species. It is therefore possible that SINEs may indeed have occupied all of the potential insertion sites in the genome of the common ancestor of E. histolytica and $E$. dispar but many of the inserted elements may have been preferentially lost in each genome as the two species diverged from each other. Indeed the differential loss of retrotransposons from specific loci might have contributed to speciation $[25,26]$. On the other hand it may be possible that SINE expansion took place after the divergence of the two species, and only a sub set of the potential insertion sites in the E. histolytica and $E$. dispar genomes are currently occupied. In that case one may expect that each of these extant genomes may possess a large number of 'empty' sites where SINEs could potentially insert in future. A hallmark of retrotransposition is the appearance of target site duplication (TSD) following the insertion of a new element. In syntenic loci an unoccupied site is expected to have one copy of the TSD which is duplicated in the occupied site. We checked for TSD sequences in E. dispar unoccupied sites corresponding to the syntenic E. histolytica occupied sites. We randomly picked 75 loci of E. histolytica, where SINE1 is absent in E. dispar and looked for matches with the TSD at each locus. At 19 loci we found very good match with the TSD sequence (matched length greater than $15 \mathrm{bp}$, and sequence identity greater than $85 \%$ ). The occurrence of close matches of TSD sequences in the syntenic loci of $E$. dispar suggests that potential empty sites may exist where future retrotransposition events could take place.

\section{Conclusions}

Our data show that the LINE-encoded endonucleases, Eh EN and Ed EN are essentially very similar, both in their kinetic properties and in their substrate sequence specificity. The DNA structural features of SINE-occupied sites in E. histolytica and E. dispar are also similar. However the elements do not insert at the same sites in the two species. Even in the $20 \%$ cases where the elements are located in the same intergenic regions in the two genomes the exact point of insertion is not the same. It is possible that, despite the Eh EN and Ed EN being very similar, the complete retrotransposition machinery consisting of the ribonucleoprotein assembly of LINE-encoded ORF1, ORF2 and the SINE transcript might function differently in the two species, thus leading to the observed differences. Since the elements are very closely related, these differences are likely to be the result of subtle changes that got established after the divergence of the two species. An experimental test of these functional changes requires the complete assembly of ORF1 and ORF2 of EdLINE1. On the other hand the possibility exists that the retrotransposition machineries in the two species are in fact identical and the observed differences are due to the stochastic nature of the insertion process. The number of potential insertion sites of these elements is likely to be large since they do not insert at specific sequences. The only known specificity of the process is that the elements in both E. histolytica and $E$. dispar insert only in intergenic regions and not 
within genes. Within intergenic regions they insert near T-rich stretches $[18,19]$. As discussed above, in this scenario one would expect to find a large number of 'empty' sites in each genome which could be targets of future insertions. This needs to be experimentally tested. A further possibility is that common insertions did indeed occur in the two genomes, but these were subsequently lost in a differential manner due to selection pressure. If the loss of SINE copies from specific locations conferred a growth advantage to either species, these elements could well have shaped the physiological evolution of the two species, including their virulence properties.

Whatever may have been the mechanisms and processes that determined the positioning of SINEs, the resultant effect is that SINEs occupy different intergenic locations in the two genomes. It is well recognized that LINEs and SINEs can modulate the expression of genes in their vicinity by providing alternative promoters, splicing and polyadenylation sites and by heterochromatinization [10-13]. The absence of SINE1 in $>80 \%$ of syntenic loci in the extant genomes of E. histolytica and $E$. dispar could result in differential expression of genes at these loci. This could profoundly influence the phenotype of the two species, which needs to be explored. Recently Lorenzi et al. [27] in their reannotation and analysis of the E. histolytica genome have listed a large number of protein families showing high association with repetitive elements. Though the top three families which are associated with TEs $100 \%$ of the time are hypothetical proteins, important known protein families are also listed. These include gal/gal Nac lectin, hsp70 BspA-like surface protein family and AIG family associated with resistance to bacteria. Further analysis of a similar nature with $E$. dispar genome will give interesting information on the possible contribution of TEs in regulating the expression of important genes that may influence pathogenesis.

\section{Additional material}

Additional file 1: Figure S1. Schematic representation of overlapping PCRs for construction of Ed EN. Sixteen sets of primers incorporating the desired mutations were designed. PCR was done using PET-Eh-EN construct as template. The $782 \mathrm{bp}$ fragment containing Ed EN domain was cloned in $\mathrm{pET30}(\mathrm{b})$ vector at the EcoRI-Notl site.

Additional file 2: Table S1. Primer sets used for the construction of Ed EN.

Additional file 3: Figure S2. Graphical representation of the syntenic region where SINE1 is present in E. histolytica but absent in E. dispar. For color code and arrows refer to figure S1-S17

Additional file 4: Figure S3-S19. Graphical representation of the syntenic region where SINE1 is present in both E. histolytica and E. dispar. Top and bottom green lines are showing the genomes of E. histolytica and $E$. dispar respectively. Red arrows show the Eh and Ed genes, and the gene orientation. Shaded black or Red (connecting) lines show the identical region within E. histolytica and E. dispar. Darkness of the shade is proportional to the \% Identity. Blue arrows show the repeat elements. SINE1 has been shown above the marked Yellow dot in both E. hitolytica and $E$. dispar in the syntenic region.

Additional file 5: Figure S20. Graphical representation of syntenic region where SINE1 is present in E. dispar but absent in E. histolytica. For color code and arrows refer to figure S1-S17.

\section{Acknowledgements}

This work was supported by a grant to SB from Indian Council of Medical Research and by fellowship from Council of Scientific and Industrial Research, India to VK and AKG.

\section{Author details}

${ }^{1}$ School of Environmental Sciences, Jawaharlal Nehru University, New Delhi 110067, India. ${ }^{2}$ School of Life Sciences, Jawaharlal Nehru University, New Delhi 110067, India.

\section{Authors' contributions}

SB proposed and designed the research, drafted the final version of the manuscript, $A B$ designed and analyzed the computational work. VK performed the experiments regarding cloning, expression, purification and assay of Ed EN. RS performed the computational work. VPY helped in performing the enzyme kinetics of Ed EN, AKG helped in the cloning of Ed EN. All authors have participated in preparing the manuscript. All authors have read and approved the final manuscript.

Received: 27 January 2011 Accepted: 25 May 2011

Published: 25 May 2011

\section{References}

1. Belancio VP, Hedges DJ, Deininger P: Mammalian non-LTR retrotransposons: for better or worse, in sickness and in health. Genome Res 2008, 18(3):343-58.

2. Ebihara M, Ohba H, Ohno SI, Yoshikawa T: Genomic organization and promoter analysis of the human nicotinic acetylcholine receptor alpha6 subunit (CHNRA6) gene: Alu and other elements direct transcriptional repression. Gene 2002, 298(1):101-8.

3. Moolhuijzen P, Kulski JK, Dunn DS, Schibeci D, Barrero R, Gojobori T, Bellgard M: The transcript repeat element: the human Alu sequence as a component of gene networks influencing cancer. Funct Integr Genomics.

4. Huntley DM, Pandis I, Butcher SA, Ackers JP: Bioinformatic analysis of Entamoeba histolytica SINE1 elements. BMC Genomics 2010, 11:321.

5. Bakre AA, Rawal K, Ramaswamy R, Bhattacharya A, Bhattacharya S: The LINEs and SINEs of Entamoeba histolytica: comparative analysis and genomic distribution. Exp Parasitol 2005, 110(3):207-213.

6. Lorenzi H, Thiagarajan M, Haas B, Wortman J, Hall N, Caler E: Genome wide survey, discovery and evolution of repetitive elements in three Entamoeba species. BMC Genomics 2008, 9:595.

7. Sharma R, Bagchi A, Bhattacharya A, Bhattacharya S: Characterization of a retrotransposon-like element in Entamoeba histolytica. Mol Biochem Parasitol 2001, 116(1):45-53.

8. Van Dellen K, Field J, Wang Z, Loftus B, Samuelson J: LINEs and SINE-like elements of the protist Entamoeba histolytica. Gene 2002, 297(1-2):229-239.

9. Shire AM, Ackers JP: SINE elements of Entamoeba dispar. Mol Biochem Parasitol 2007, 152(1):47-52.

10. Faulkner GJ, Carninci P: Altruistic functions for selfish DNA. Cell Cycle 2009, 8(18):2895-900.

11. Chow JC, Ciaudo C, Fazzari MJ, Mise N, Servant N, Glass JL, Attreed M, Avner P, Wutz A, Barillot E, Greally JM, Voinnet O, Heard E: LINE-1 activity in facultative heterochromatin formation during $X$ chromosome inactivation. Cell 2010, 141(6):956-69.

12. Lev-Maor G, Sorek R, Shomron N, Ast G: The birth of an alternatively spliced exon: 3' splice-site selection in Alu exons. Science 2003, 300(5623):1288-91.

13. Ostertag EM, Kazazian HH Jr: Biology of mammalian L1 retrotransposons. Annu Rev Genet 2001, 35:501-538. 
14. Willhoeft $\mathrm{U}$, Buss $\mathrm{H}$, Tannich E: The abundant polyadenylated transcript 2 DNA sequence of the pathogenic protozoan parasite Entamoeba histolytica represents a nonautonomous non-long-terminal-repeat retrotransposon-like element which is absent in the closely related nonpathogenic species Entamoeba dispar. Infect immun 2002, 70(12):6798-6804.

15. Luan DD, Korman MH, Jakubczak JL, Eickbush TH: Reverse transcription of R2Bm RNA is primed by a nick at the chromosomal target site: a mechanism for non-LTR retrotransposition. Cell 1993, 72(4):595-605.

16. McGinnis S, Madden TL: BLAST: at the core of a powerful and diverse set of sequence analysis tools. Nucleic Acids Res 2004, 32 Web Server: W20-25.

17. Nix DA, Eisen MB: GATA: a graphic alignment tool for comparative sequence analysis. BMC Bioinformatics 2005, 6:9.

18. Mandal PK, Rawal K, Ramaswamy R, Bhattacharya A, Bhattacharya S: Identification of insertion hot spots for non-LTR retrotransposon: computational and biochemical application to Entamoeba histolytica. Nucleic Acids Res 2006, 34(20):5752-63.

19. Mandal PK, Bagchi A, Bhattacharya A, Bhattacharya S: An Entamoeba histolytica LINE / SINE pair inserts at common target sites cleaved by the restriction enzyme-like LINE encoded endonuclease. Eukaryot Cell 2004, 3(1):170-179.

20. Yadav VP, Mandal PK, Rao DN, Bhattacharya S: Characterization of the restriction enzyme-like endonuclease encoded by the Entamoeba histolytica non-long terminal repeat retrotransposon EhLINE1. FEBS J 2009, 276(23):7070-7082.

21. Sambrook J, Russell DW: Molecular cloning: a laboratory manual. 3 edition. Cold Spring Harbor Laboratory Press, Cold Spring Harbor, N.Y; 2001.

22. Pingoud A, Alves J, Geiger R: Restriction enzymes. Methods Mol Biol 1993, 16:107-200.

23. Silberman JD, Clark CG, Diamond LS, Sogin ML: Phylogeny of the genera Entamoeba and Endolimax as deduced from small-subunit ribosomal RNA sequences. Mol Biol Evol 1999, 16(12):1740-1751.

24. Diamond LS, Clark CG: A redescription of Entamoeba histolytica Schaudinn, 1903 (Emended Walker, 1911) separating it from Entamoeba dispar Brumpt, 1925. J Eukaryot Microbiol 1993, 40(3):340-344.

25. Böhne A, Brunet F, Galiana-Arnoux D, Schultheis C, Volff JN: Transposable elements as drivers of genomic and biological diversity in vertebrates. Chromosome Res 2008, 16(1):203-215.

26. Jurka J, Kapitonov W, Kohany O, Jurka MV: Repetitive sequences in complex genomes: structure and evolution. Annu Rev Genomics Hum Genet 2007, 8:241-259.

27. Lorenzi HA, Puiu D, Miller JR, Brinkac LM, Amedeo P, Hall N, Caler EV: New assembly, reannotation and analysis of the Entamoeba histolytica genome reveal new genomic features and protein content information. PLoS Negl Trop Dis 2010, 4(6):e716.

doi:10.1186/1471-2164-12-267

Cite this article as: Kumari et al:: Differential distribution of a SINE element in the Entamoeba histolytica and Entamoeba dispar genomes: Role of the LINE-encoded endonuclease. BMC Genomics 2011 12:267.

\section{Submit your next manuscript to BioMed Central and take full advantage of:}

- Convenient online submission

- Thorough peer review

- No space constraints or color figure charges

- Immediate publication on acceptance

- Inclusion in PubMed, CAS, Scopus and Google Scholar

- Research which is freely available for redistribution 